\title{
Mixed pulmonary infection with Nocardia, Candida, methicillin-resistant Staphylococcus aureus, and group D Streptococcus species
}

\author{
Joseph Vassallo, Alfred Caruana Galizia, Paul Cuschieri
}

\begin{abstract}
Summary
A 67-year-old man on prednisolone and azathioprine for ulcerative colitis, developed severe pneumonia due to Nocardia otitidis caviarum, methicillinresistant Staphylococcus aureus, a group D Streptococcus and Candida albicans. The patient responded well to aggressive antimicrobial therapy.
\end{abstract}

Keywords: pneumonia, Nocardia otitidis caviarum, Staphylococcus aureus, Streptococcus, Candida albicans

A 67-year-old man with a long-standing history of ulcerative colitis, on azathioprine and high dose prednisolone, was admitted with a oneweek history of fever and productive cough. His general practitioner had previously given him a course of oral cefuroxime axetil, $250 \mathrm{mg}$ bid, with no effect.

On admission he had clinical and radiological features of left lower lobe pneumonia. His initial white cell count was $10.1 \times 10^{9}$ cells $/$. He was started on intravenous ceftazidime, $2 \mathrm{~g}$ 8 hourly, and gentamicin, $80 \mathrm{mg} 8$ hourly, without any response and his clinical and radiological condition continued to deteriorate. A direct Gram stain, a Ziehl-Neelsen and a modified Ziehl-Neelsen stain, performed on freshly expectorated sputum, yielded packed fields of Gram-positive cocci, numerous yeasts and hyphae, and weakly acidfast, Gram-positive, branching filaments. A presumptive diagnosis of a mixed infection due to Nocardia, Candida and Staphylococcus and/or Streptococcus was made and the antimicrobial therapy was changed to intravenous imipenem $500 \mathrm{mg} 6$ hourly, co-trimoxazole, $120 \mathrm{mg} / \mathrm{kg}$ daily in four divided doses, and fluconazole, $50 \mathrm{mg}$ bid. The azathioprine was discontinued and the dose of prednisolone was gradually tapered down. His condition improved but sputum culture yielded a methicillin-resistant Staphylococcus aureus (MRSA) which was resistant to cloxacillin, gentamicin, erythromycin, co-trimoxazole, ciprofloxacin and imipenem, but sensitive to vancomycin, fusidic acid, clindamycin and netilmicin. The sputum specimens were all cultured without significant delays and were rapidly digested at room temperature with $\mathrm{N}$-acetyl cysteine, to a final dilution of 1 in 200 before culture, effectively diluting out commensals.

A group D Streptococcus which was sensitive to vancomycin, fusidic acid and ampicillin was cultured. The latter organism is increasingly being identified as a pathogen in immunocompromised patients. Candida albicans and Nocardia species were also cultured. The latter was later identified as Nocardia otitidis caviarum.

Subsequently the patient was started on additional parenteral vancomycin and fusidic acid and his condition improved markedly as assessed by repeated clinical and radiological examinations and laboratory investigation. The latter included regular Gram staining and culturing of the sputum. The Nocardia organisms were detectable in the sputum on direct Gram stains for up to 10 days after starting effective therapy. Vancomycin was stopped after eight days of therapy. Fluconazole, fusidic acid, imipenem and intravenous co-trimoxazole were given for 14 days, while the latter preparation was changed to oral form and continued for a further eight weeks at a dose of $960 \mathrm{mg}$ bid. The patient has remained asymptomatic since then.

\section{Discussion}

To our knowledge this is the first reported case of multiple pulmonary infection of such magnitude involving Nocardia otitidis caviarum, and having a favourable outcome. We believe that all the organisms cultured, played a pathogenic role. The presence of large numbers of fungal hyphae support a pathogenic role for Candida, while the presence of large numbers of MRSA and group D Streptococcus similarly supports a pathogenic role for these organisms. The clinical condition of the patient indicated aggressive antimicrobial therapy against all these organisms. We believe that this contributed to the rapid improvement in the patient's condition, as did the early discontinuation of azathioprine.

Nocardia species are a group of aerobic Gram-positive branching, filamentous organisms which cause an acute or chronic, often disseminated disease. They are important members of the soil microflora worldwide. Nocardia can infect any individual but is much more common in patients with impaired immunity (box 1). It is not a rare condition, though it is not given prominent attention in the medical literature. It can be easily missed if the index of suspicion is low as it can resemble more common diseases, such as tumours and 


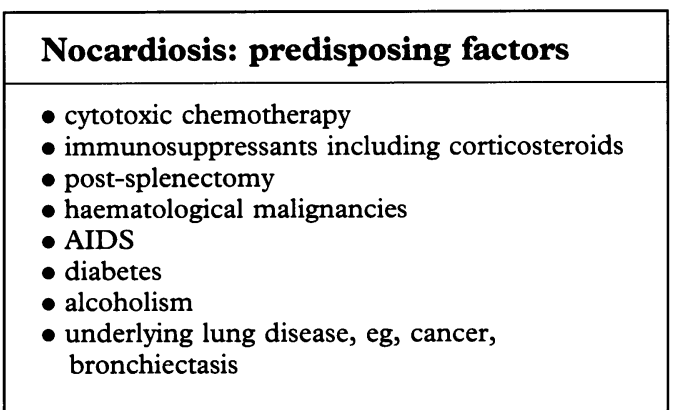

Box 1

other granulomatous diseases. ${ }^{1}$ Most cases begin as pulmonary infection and disseminate haematogenously, with abscess formation in the brain, kidneys, bones, etc. Infection may also start as skin or subcutaneous abscess.

Several species have been documented to be pathogenic in man. These are $N$ asteroides, $N$ brasiliensis, $N$ farcinica, $N$ otitidis caviarum, $N$ nova and $N$ transvalensis. ${ }^{2}$ The diagnosis is made by culture of the organism. However, as this can take weeks, therapy should be started on microscopic morphology. A chest X-ray is useful in narrowing the differential diagnosis but is nonspecific (box 2). ${ }^{3}$

Sputum examination is diagnostic in only a third of cases, and although oropharyngeal colonisation can occur, isolation of Nocardia from the sputum of an immunocompromised host should be regarded as pathogenic. It is imperative that in immunocompromised patients with lung infiltrates of uncertain causation, bronchoscopy should be performed in conjunction with BAL or even open lung biopsy to get a diagnosis. The organism can also sometimes be isolated from blood cultures.

1 Curry WA. Human nocardiosis - a clinical review with selected case reports. Arch Intern Med 1980; 140: 818-26. 2 Beaman BL, Beaman L. Nocardia species: host-parasite relationships. Clin Microbiol Rev 1994; 7: 213-64.

3 Feigin DS. Nocardiosis of the lung: chest radiographic findings in 21 cases. Radiology 1986; 159: 9-14.

4 Gutmann L, Goldstein FW, Kitzis MD, Hautefort B, Darmon C, Acar JF. Susceptibility of Nocardia asteroides to 46 antibiotics, including $22 \beta$-lactams. Antimicrob Agents Chemother 1983; 23: 248-51.

\begin{tabular}{|l|}
\hline Nocardiosis: chest X-ray findings \\
\hline - solitary pulmonary mass \\
- pulmonary infiltrates \\
- pleural thickening \\
- chest wall involvement \\
\hline
\end{tabular}

Box 2

In vitro they show susceptibility to several antibiotics including sulphonamides, co-trimoxazole, imipenem, amikacin, minocycline, fusidic acid, ceftriaxone, cefotaxime, and coamoxyclav. ${ }^{4}$ Resistance is, however, common, especially with $N$ farcinica.

The standard therapy for nocardiosis is cotrimoxazole; the combination of amikacin and imipenem is usually reserved for resistant strains or for patients allergic to sulpha drugs. Minocycline and co-amoxyclav are alternative medications. ${ }^{5}$ It is usually recommended that treatment be continued for three to six months in mild infections and for six to 12 months in severe infections, because of the risk of relapse. Patients who are on steroids or other immunosuppressants should have the dose of the steroid tapered and the immunosuppressant drug should be stopped if possible. In this case 10 weeks of treatment proved adequate. This could possibly be due to the intensive initial therapy; imipenem, co-trimoxazole and fusidic acid all show activity against Nocardia. Further investigations to study the efficacy of short-course therapy is warranted. This would improve patient compliance and reduce toxicity.

We would like to thank $\mathrm{Mr} M D$ Yates, Principal Microbiologist at the Public Health Laboratory of Dulwich Hospital, London, for his help in identifying our Nocardia isolate.

5 Thomas CF Jr, Jones TK, Edson RS. A 74-year-old woman with dyspnoea, fever, and cough. Mayo Clin Proc 1995; 70: $397-400$. 